\title{
RIESZ-NÁGY SINGULAR FUNCTIONS REVISITED
}

\author{
JAUME PARADÍS, PELEGRÍ VIADER, AND LLUÍS BIBILONI
}

\begin{abstract}
In 1952 F. Riesz and Sz.-Nágy published an example of a monotonic continuous function whose derivative is zero almost everywhere, that is to say, a singular function. Besides, the function was strictly increasing. Their example was built as the limit of a sequence of deformations of the identity function. As an easy consequence of the definition, the derivative, when it existed and was finite, was found to be zero. In this paper we revisit the Riesz-Nágy family of functions and we relate it to a system for real number representation which we call $(\tau, \tau-1)$-expansions. With the help of these real number expansions we generalize the family. The singularity of the functions is proved through some metrical properties of the expansions used in their definition which also allows us to give a more precise way of determining when the derivative is 0 or infinity.
\end{abstract}

\section{INTRODUCTION}

At the beginning of the XX-th century singular functions were considered quite pathological and remained so for a long time. In those times, besides the usual Cantor-Lebesgue's type of functions - constant on the complement of a perfect set of measure zero-, examples of a different sort of a singular function were not easy to provide. To think of a strictly increasing function that were at the same time singular, was simply difficult to accept. One of the simplest cases of a strictly increasing continuous singular function was Minkowski's "fragefunktion", ? $(x)$. Its purpose however was a completely arithmetical one: to obtain an enumeration of the irrational quadratics [11]. Its construction was rather simple as it was essentially geometrical in nature. It was much later that its singularity was proved. This was done by Denjoy in 1932 [4] and [5], though the classical reference is Salem with his paper [17]. Minkowski's function has been extensively studied and generalized (Denjoy [6], [10], Tichy [20], Ramharter [13], Girgensohn [7]). In [21] and [12] the authors contributed to the subject proving that ?' $(x)$, when it existed and was finite, had to be zero and obtained conditions that allowed to separate null from infinite derivatives.

Another family of strictly increasing singular functions was popularized by Riesz and Sz.-Nágy who published them in 1952 [16, p. 48-49]. Their example was built as the limit of a sequence of deformations of the identity function. It depended on a parameter $0<t<1$. As an easy consequence of the definition, the derivative, when it existed, was found to be a Riesz-product: $\prod_{k}\left(1+\varepsilon_{k} t\right),(\varepsilon= \pm 1)$ which can only be 0 , infinity or indeterminate.

In fact, Riesz-Nágy functions had already been considered before. Hellinger mentions them in his doctoral thesis in 1907 [8] and other authors work with the family: Billingsley [1, pp. 35-37] and [2, pp. 407-409], de Rahm [3], Reese [14]; or have even generalized it: Salem [17], Hewitt and Stromberg [9, p. 278-282].

Date: January 2006.

1991 Mathematics Subject Classification. Primary 26A30; Secondary 11K55.

Key words and phrases. Singular functions, metric number theory. 
In 1978 Lajos Takács published a short note in the MonThLy [19] providing a closed form definition of a family of increasing continuous singular functions. The main merit of Takács' paper is the realization that his family of functions is precisely the Riesz-Nágy family.

In this paper we revisit the Riesz-Nágy family of functions and we show how Takács closed form relates to a new system for real number representation which we call $(\tau, \tau-1)$-expansions. In section 2 , we present these expansions in some detail as their metrical properties will be essential for our purposes. Specifically, in section 3 we prove the singularity of the family using some of these metrical properties. Besides, we obtain metrical conditions that allow us to separate the null from the infinite derivative. In section 4 with the help of $(\tau, \tau-1)$-expansions we generalize the family and obtain similar conditions for the null and infinite derivatives.

\section{ON $(\tau, \tau-1)$-EXPANSIONS}

Given $\tau \in \mathbb{R}, \tau>1$, let us consider the unit interval $[0,1)$ and its partition into two parts by $1 / \tau$ :

$$
[0,1)=\left[0, \frac{1}{\tau}\right) \bigcup\left[\frac{1}{\tau}, 1\right) .
$$

Any real number $x \in[0,1)$ has the following expression:

$$
x= \begin{cases}\frac{1}{\tau} \cdot x_{1} & \text { if } \quad x<\frac{1}{\tau} \\ \frac{1}{\tau}+\frac{\tau-1}{\tau} \cdot x_{1} & \text { if } \quad x \geq \frac{1}{\tau}\end{cases}
$$

where, in both cases, $x_{1} \in[0,1)$.

Iteration of the previous process, leads us to an expansion for $x$ in powers of the numbers $\frac{1}{\tau}$ and $\frac{\tau-1}{\tau}$ :

$$
x=\sum_{i=1}^{\infty} \varepsilon_{i} \frac{1}{\tau^{i}}(\tau-1)^{\sum_{j=1}^{i-1} \varepsilon_{j}}, \quad \varepsilon_{i} \in\{0,1\} .
$$

If $x_{0}:=x$, the algorithm, for $n \geq 1$ is the following:

$$
x_{n}:=\left\{\begin{array}{ll}
\tau x_{n-1} & \text { if } \quad x_{n-1}<\frac{1}{\tau} \\
\frac{\tau x_{n-1}-1}{\tau-1} & \text { if } \quad x_{n-1} \geq \frac{1}{\tau}
\end{array} \quad \rightarrow \quad \varepsilon_{n}= \begin{cases}0 & \text { if } \quad x_{n}<\frac{1}{\tau} \\
1 & \text { if } \quad x_{n} \geq \frac{1}{\tau}\end{cases}\right.
$$

This expansion is a particular case of an $f$-expansion as defined by Rényi [15] or a $\bar{\beta}$-expansion with $\bar{\beta}=\left(\tau, \frac{\tau}{\tau-1}\right)$ of Shiokawa [18].

Now, every $x \in[0,1)$ can be represented through its 'digits', $\left\langle\varepsilon_{i}\right\rangle_{i \in \mathbb{Z}^{+}}$. It is readily seen that, in case $x$ has a finite expansion as a consequence of $x_{n+1}=0$, $x=\left\{\varepsilon_{1}, \cdots, \varepsilon_{n}\right\}$, where $\varepsilon_{n}=1$, then $x$ has also a non terminating equivalent expansion simply by changing the digit $\varepsilon_{n}$ to 0 and making $\varepsilon_{n+k}=1, k=1,2, \ldots$. This is a direct consequence of

$$
1=\sum_{i=1}^{\infty} \frac{(\tau-1)^{i-1}}{\tau^{i}} .
$$

The right-shift, $T$, induced by algorithm 2.2 on $[0,1)$ has the graph that Figure 1 shows.

Definition 1. We will say that $x$ is normal to base $(\tau, \tau-1)$ if the sequence $\left\langle x_{n}\right\rangle$ is uniformly distributed in $[0,1)$. 


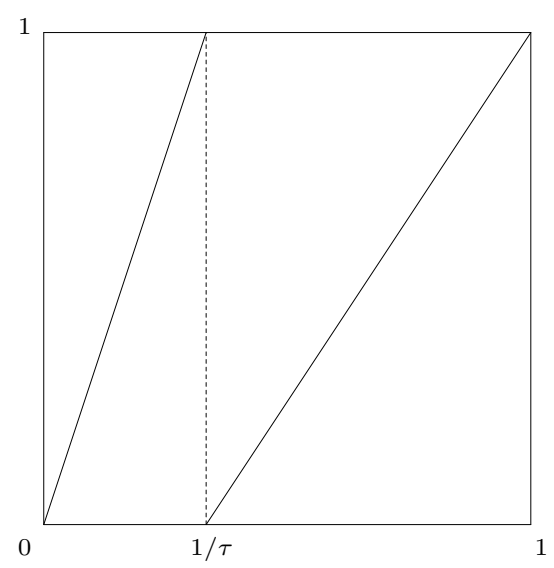

FIGURE 1. $(\tau, \tau-1)$-right-shift

The right shift, $T x_{n}=x_{n+1}$, attached to our algorithm, trivially preserves Lebesgue's measure in $[0,1)$ and is ergodic. This last fact was proved by Rényi in [15] for a wide class of $f$-expansions with 'independent digits' (meaning that any sequence of digits $\left\langle\varepsilon_{i}\right\rangle$ is admissible, that is to say, it corresponds to the $f$-expansion of a real number). As a result, the orbit, $\left\langle T^{n} x\right\rangle$ of almost all $x \in[0,1)$ is uniformly distributed, or, what amounts to the same according to Definition 1, almost all numbers in $[0,1)$ are normal to base $(\tau, \tau-1)$.

For these normal numbers, we have, on the one hand

$$
\lim _{n \rightarrow \infty} \frac{\sharp\left\{\varepsilon_{i}(x)=1, i=1, \ldots, n\right\}}{n}=\frac{\tau-1}{\tau},
$$

and on the other hand, the frequency of any given finite sequence $\varepsilon_{1}, \ldots, \varepsilon_{k}$ is

$$
\left(\frac{\tau-1}{\tau}\right)^{r}\left(\frac{1}{\tau}\right)^{k-r}
$$

where $r$ is the number of 1 's in the given sequence,

$$
r=\sum_{i=1}^{k} \varepsilon_{i}
$$

2.1. Making blocks. We can reach the same results of the previous section in a more compact form using as a partition of $[0,1)$ the one whose division points are the different powers of $1 / \tau$ :

$$
(0,1)=\bigcup_{i=1}^{\infty}\left[\frac{1}{\tau^{i}}, \frac{1}{\tau^{i-1}}\right) .
$$

Any $x \in[0,1)$ will belong to one of the above intervals, $\left[\tau^{-a_{1}}, \tau^{-\left(a_{1}-1\right)}\right)$ and we will be able to write

$$
x:=x_{1}=\frac{1}{\tau^{a_{1}}}+\left(\frac{1}{\tau^{a_{1}-1}}-\frac{1}{\tau^{a_{1}}}\right) \cdot x_{2}, \quad x_{2} \in[0,1),
$$

which is the same as

$$
x:=x_{1}=\frac{1}{\tau^{a_{1}}}+\frac{\tau-1}{\tau^{a_{1}}} x_{2} .
$$

Iterating the procedure, we obtain the expansion,

$$
x=\frac{1}{\tau^{a_{1}}}+\frac{\tau-1}{\tau^{a_{1}}} \cdot \frac{1}{\tau^{a_{2}}}+\cdots+\frac{(\tau-1)^{n-1}}{\tau^{a_{1}+\cdots+a_{n}}}+\cdots, \quad a_{i} \in \mathbb{Z}^{+} .
$$


As before, to any finite expansion, there corresponds a non terminating expansion owing to the identity

$$
\frac{1}{\tau^{k}}=\sum_{i=1}^{\infty} \frac{(\tau-1)^{i-1}}{\tau^{k+i}} .
$$

The algorithm that leads to (2.4) is the following:

$$
\left\{\begin{aligned}
x_{1} & :=x ; \\
a_{n} & :=\left[\log _{\tau}\left(\frac{1}{x_{n}}\right)\right]+1, \quad n=1,2, \ldots ; \\
x_{n} & :=\frac{x_{n-1} \tau^{a_{n-1}}-1}{\tau-1} \quad n=2,3, \ldots ;
\end{aligned}\right.
$$

Here $[z]$ denotes the usual integer part of $z$.

If $s_{n}=a_{1}+\cdots+a_{n}$, then expansion (2.4) can be written

$$
x=\sum_{i=1}^{\infty} \frac{(\tau-1)^{i-1}}{\tau^{s_{i}}}, \quad 1 \leq s_{1}<s_{2}<\cdots<s_{n}<\cdots .
$$

In terms of these $s_{n}$, the result (2.3) becomes

Theorem 2.1. If $x$ is normal to base $(\tau, \tau-1)$, we have

$$
\lim _{n \rightarrow \infty} \frac{s_{n}(x)}{n}=\frac{\tau}{\tau-1} .
$$

There are two values of $\tau$ that give rise to interesting expansions. For $\tau=2$, we get the familiar dyadic system. For $\tau=\Phi=(1+\sqrt{5}) / 2$, the golden number verifying $\Phi-1=1 / \Phi$, we get the expansion

$$
x=\sum_{i=1}^{\infty} \frac{1}{\Phi^{s_{i}}} \frac{1}{\Phi^{i-1}}=\sum_{i=1}^{\infty} \frac{1}{\Phi^{s_{i}+i-1}}, \quad 1 \leq s_{1}<s_{2}<\cdots
$$

or, in a more compact form,

$$
x=\sum_{i=1}^{\infty} \frac{1}{\Phi^{k_{i}}}, \quad k_{1} \geq 1, k_{i+1}>k_{i}+1 .
$$

This is an expansion of $x$ as a sum of reciprocal powers of $\Phi$ with no two consecutive powers and coincides exactly with Rényi's $\beta$-expansions for $\beta=\Phi[15]$.

\section{TAKÁCS' FUNCTION AND $(\tau, \tau-1)$-EXPANSIONS}

The results of the previous section are closely related to a function exhibited by Takács, [19]:

$$
F:[0,1] \longrightarrow[0,1]
$$

with $F(0)=0, F(1)=1$ and if $x=\sum_{r=1}^{\infty} 2^{-a_{r}}$, then

$$
F(x)=\sum_{r=1}^{\infty} \frac{\rho^{r-1}}{(1+\rho)^{a_{r}}}
$$

where $\rho$ is a positive real number. If we take $\rho=\tau-1$ we immediately see that the definition of $F(x)$ corresponds to one of our $(\tau, \tau-1)$-expansions:

$$
F(x)=\sum_{r=1}^{\infty} \frac{(\tau-1)^{r-1}}{\tau^{a_{r}}}
$$

$\tau$ a given real number, $\tau>1$. 
Takács' function, proved to be an increasing continuous singular function, that is to say, with a vanishing derivative almost everywhere in $[0,1)$. Takács, in the mentioned paper, does not explain how he arrived at his function.

As we have already mentioned the real importance of Takács paper is the realization that part of his family of functions, for $0<\rho<1$, was precisely the family of singular functions introduced by Riesz and Nágy in [16, p. 48-49]. To be more precise, the relationship between Ries-Nágy parameter $t(0<t<1)$, Takács $\rho(\rho>0)$ parameter and our $\tau(\tau>1)$ is:

$$
t=\frac{1-\rho}{1+\rho}=\frac{1-\tau}{\tau}
$$

where $0<\rho<1$ and $1<\tau<2$. Takács (and other authors mentioned above) proved the singularity of $F(x)$ by proving that, for $\tau>1$, whenever $F^{\prime}(x)$ existed and was finite it had to be 0 . Then, as by Lebesgue's Theorem any monotone function has a finite derivative except possibly on a set of measure zero, $F^{\prime}(x)=0$ almost everywhere on $[0,1)$.

Thanks to the identification of Takács function with $(\tau, \tau-1)$-expansions, we are going to carry the study of its singularity a little further. Let us change the function's name to $\Phi_{2, \tau}$ for reasons that will be explained presently.

Theorem 3.1. If $x=\sum_{j=1}^{\infty} 2^{-a_{j}}, 1 \leq a_{1}<a_{2}<\cdots$, is simply normal to base 2 in the sense of Borel, and $\Phi_{2, \tau}^{\prime}(x)$ exists and is finite then $\Phi_{2, \tau}^{\prime}(x)=0$.

Proof. We give a proof of this result which is somewhat different to the one given by Riesz and Nágy as we will need the procedure used in it later.

Let $x=\sum_{j=1}^{\infty} 2^{-a_{j}} \in[0,1)$ and let $n$ be such that $a_{n+1}>a_{n}+1$. Such a value of $n$ must exist unless $x$ is a finite decimal. A straddling interval for $x$ will be

$$
\sum_{j=1}^{n} \frac{1}{2^{a_{j}}}<x<\sum_{j=1}^{n} \frac{1}{2^{a_{j}}}+\frac{1}{2^{a_{n}+1}} .
$$

As $\Phi_{2, \tau}$ is strictly increasing, the images corresponding to the endpoints will satisfy:

$$
\sum_{j=1}^{n} \frac{(\tau-1)^{j-1}}{\tau^{a_{j}}}<\Phi_{2, \tau}(x)<\sum_{j=1}^{n} \frac{(\tau-1)^{j-1}}{\tau^{a_{j}}}+\frac{(\tau-1)^{n}}{\tau^{a_{n}+1}} .
$$

If $\Phi_{2, \tau}^{\prime}(x)$ exists, it will have to verify

$$
\begin{aligned}
\Phi_{2, \tau}^{\prime}(x)=\lim _{n \rightarrow \infty} \frac{(\tau-1)^{n} / \tau^{a_{n}+1}}{1 / 2^{a_{n}+1}} & = \\
=\lim _{n \rightarrow \infty}(\tau-1)^{n} \cdot\left(\frac{2}{\tau}\right)^{a_{n}+1} & =\lim _{n \rightarrow \infty}\left[(\tau-1)\left(\frac{2}{\tau}\right)^{a_{n} / n}\right]^{n} .
\end{aligned}
$$

If $x$ is simply normal to base 2 in the sense of Borel, then we will have $\lim _{n \rightarrow \infty} a_{n} / n=$ 2 , and consequently the base of the power between brackets in (3.2) will tend to $4(\tau-1) / \tau^{2}$, and the limit will be 0 if that base is less than 1 . Now,

$$
\frac{4(\tau-1)}{\tau^{2}}<1 \Longleftrightarrow 4 \tau-4<\tau^{2} \Longleftrightarrow(\tau-2)^{2}>0 .
$$

This last inequality is always verified if $\tau \neq 2$ (which is unavoidable as $\Phi_{2,2}=$ Id).

The trick in the proof is to have a base in the power in (3.2) tending to a number less than one. With this in mind, let us carry Theorem 3.1 a little further:

Theorem 3.2. Let $K=K(\tau)=\frac{-\log (\tau-1)}{\log (2 / \tau)}$. If the dyadic expansion of $x \in[0,1)$ is $x=\sum_{j=1}^{\infty} 2^{-a_{j}}$, then we have: 
i) Case $1<\tau<2$. If there exists a value $k$ such that

$$
\limsup _{n \rightarrow \infty} \frac{a_{n}}{n} \leq k<K
$$

then if $\Phi_{2, \tau}^{\prime}(x)$ exists, it has to be 0 .

If

$$
\liminf _{n \rightarrow \infty} \frac{a_{n}}{n} \geq k>K
$$

then, if $\Phi_{2, \tau}^{\prime}(x)$ exists in a wide sense, it has to be infinite.

ii) Case $\tau>2$. If there exists a value $k$ such that

$$
\liminf _{n \rightarrow \infty} \frac{a_{n}}{n} \geq k>K
$$

then if $\Phi_{2, \tau}^{\prime}(x)$ exists, it has to be 0 .

If

$$
\limsup _{n \rightarrow \infty} \frac{a_{n}}{n} \leq k<K
$$

then, if $\Phi_{2, \tau}^{\prime}(x)$ exists in a wide sense, it has to be infinite.

Proof. It is seen at once that, for all $\tau>1, K(\tau)>1$.

Case $1<\tau<2$. If $\limsup _{n \rightarrow \infty} a_{n} / n \leq k<K$, there exists a $n_{0}$ such that for $n>n_{0}$ we have $a_{n} / n \leq k<K$. For such $n$,

$$
(\tau-1)\left(\frac{2}{\tau}\right)^{a_{n} / n} \leq(\tau-1)\left(\frac{2}{\tau}\right)^{k}<(\tau-1)\left(\frac{2}{\tau}\right)^{K}=1 .
$$

Thus the limit in (3.2) is 0 . On the other hand, if $\liminf _{n \rightarrow \infty} a_{n} / n \geq k>K$, there exists a $n_{0}$ such that for $n>n_{0}$ we have $a_{n} / n \geq k>K$. For such $n$,

$$
(\tau-1)\left(\frac{2}{\tau}\right)^{a_{n} / n} \geq(\tau-1)\left(\frac{2}{\tau}\right)^{k}>(\tau-1)\left(\frac{2}{\tau}\right)^{K}=1 .
$$

Thus the limit in $(3.2)$ is $\infty$.

A similar reasoning leads to the second part of the theorem involving the case $\tau>2$.

Figure 2 summarizes these results in a graphical way.

\section{Generalization of Riesz-Nágy-Takács functions.}

The binary system coincides with our $(\tau, \tau-1)$-expansion for $\tau=2$. In the definition of $\Phi_{2, \tau}$ then, nothing can stop us from replacing the binary system used to expand $x$ for a $(\alpha, \alpha-1)$-expansion. We obtain the function denoted by $\Phi_{\alpha, \tau}(x)$.

Definition 2. Given $\alpha, \tau>1$ and $x \in[0,1)$, if

$$
x=\sum_{j=1}^{\infty} \frac{(\alpha-1)^{j-1}}{\alpha^{a_{j}}} \quad \text { with } \quad 1 \leq a_{1}<\cdots<a_{n}<\cdots,
$$

we define

$$
\Phi_{\alpha, \tau}(x)=\sum_{j=1}^{\infty} \frac{(\tau-1)^{j-1}}{\tau^{a_{j}}} .
$$

Of course, if $\alpha=\tau, \Phi_{\tau, \tau}$ is just the identity. The interesting case will then be the case $\alpha \neq \tau$.

We will prove that this new family consists of functions which are also singular and that verify a theorem similar to Theorem 3.2. 


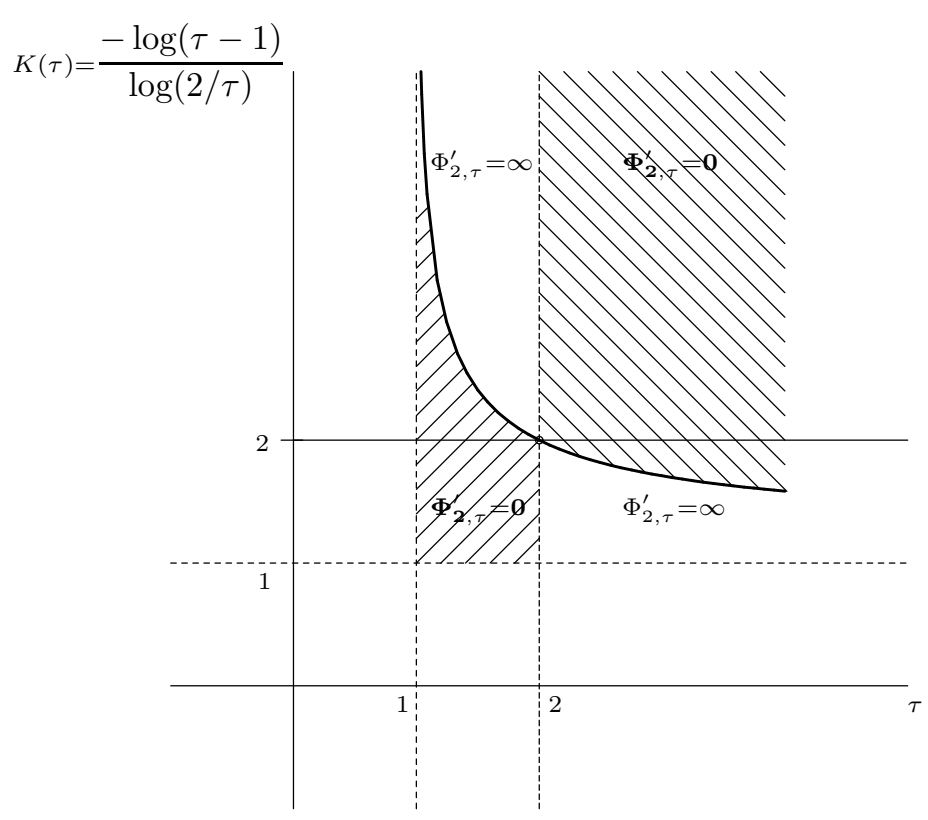

Figure 2. $K(\tau)$ and $\Phi_{2, \tau}^{\prime}$ values

As before, it is easily seen that whenever the derivative of $\Phi_{\alpha, \tau}(x)$ exists, it has to coincide with the limit

$$
\Phi_{\alpha, \tau}^{\prime}(x)=\lim _{n \rightarrow \infty} \frac{(\tau-1)^{n}}{\tau^{a_{n}+1}} \cdot \frac{\alpha^{a_{n}+1}}{(\alpha-1)^{n}}=\lim _{n \rightarrow \infty}\left(\frac{\tau-1}{\alpha-1} \cdot \frac{\alpha^{\frac{a_{n}}{n}}}{\tau^{\frac{a_{n}}{n}}}\right)^{n} .
$$

This limit will be zero if the upper limit of the base tends to a constant less than 1 , that is to say,

$$
\limsup _{n \rightarrow \infty} \frac{\tau-1}{\alpha-1} \cdot\left(\frac{\alpha}{\tau}\right)^{\frac{a_{n}}{n}} \leq k<1
$$

Let us now prove the singularity of these functions seeing that for all normal numbers to base $(\alpha, \alpha-1)$ where $\Phi_{\alpha, \tau}^{\prime}(x)$ exists, it has to vanish necessarily.

Theorem 4.1. If $x$ is normal to base $(\alpha, \alpha-1)$, then it verifies condition (4.1).

\section{Proof}

By Theorem (2.1) if $x$ is normal to base $(\alpha, \alpha-1)$, it verifies:

$$
\lim _{n \rightarrow \infty} \frac{a_{n}}{n}=\frac{\alpha}{\alpha-1} .
$$

We must now see that for all $\alpha, \tau>1, \alpha \neq \tau$ we have the following inequality

$$
H(\alpha, \tau)=\frac{\tau-1}{\alpha-1}\left(\frac{\alpha}{\tau}\right)^{\frac{\alpha}{\alpha-1}}<1 .
$$

This can be done with a little bit of calculus. The singular points of $H(\alpha, \tau)$ are precisely those along the line $\alpha=\tau$. At these points the function is concave, ensuring that the function attains a local maximum. There are no other singular points and the values at the frontier of the domain, $\{(a, 1): a>1\} \cup\{(1, t): t>1\}$ tend to 0 . Consequently the value $H(\alpha, \alpha)=1$ attained at the points of the line $\alpha=\tau$ is a global maximum. 
As we have $\alpha \neq \tau$ that means that $H(\alpha, \tau)<1$ for different bases, and that ensures the validity of the theorem.

But the same path we followed before can be taken now, and we can prove the following theorem whose proof we omit as it follows the same ideas than that of Theorem 3.2:

Theorem 4.2. Let $K=K(\alpha, \tau)=\frac{\log \left(\frac{\alpha-1}{\tau-1}\right)}{\log (\alpha / \tau)}$. If $x \in[0,1)$ has the $(\alpha, \alpha-1)$ expansion $x=\sum_{j=1}^{\infty}(\alpha-1)^{j-1} \alpha^{-a_{j}}$, then we have:

i) Case $1<\tau<\alpha$. If there exists a value $k$ such that

$$
\limsup _{n \rightarrow \infty} \frac{a_{n}}{n} \leq k<K
$$

then if $\Phi_{\alpha, \tau}^{\prime}(x)$ exists, it has to be 0 . If

$$
\liminf _{n \rightarrow \infty} \frac{a_{n}}{n} \geq k>K
$$

then, if $\Phi_{\alpha, \tau}^{\prime}(x)$ exists in a wide sense, it has to be infinite.

ii) Case $\tau>\alpha>1$. If there exists a value $k$ such that

$$
\liminf _{n \rightarrow \infty} \frac{a_{n}}{n} \geq k>K
$$

then if $\Phi_{\alpha, \tau}^{\prime}(x)$ exists, it has to be 0. If $\limsup _{n \rightarrow \infty} \frac{a_{n}}{n} \leq k<K$ then, if $\Phi_{\alpha, \tau}^{\prime}(x)$ exists in a wide sense, it has to be infinite.

\section{CONCLUding REMARKS}

We have shown how the Riesz-Nágy family of singular functions can be seen as the 'confrontation' of two systems for real number representation:

$$
\begin{aligned}
x=\sum_{r=1}^{\infty} 2^{-a_{r}} & {[\text { binary }] } \\
\Phi_{2, \tau}(x)=\sum_{r=1}^{\infty} \frac{(\tau-1)^{r}}{\tau^{a_{r}}} & {[(\tau, \tau-1) \text {-expansion }] . }
\end{aligned}
$$

That is perfectly in tune with the now familiar expression for Minkowski's ? $(x)$ function that 'confronts' the continued fraction expansion of a real number with what can be called the alternated binary system:

$$
\begin{gathered}
x=\frac{1}{a_{1}+\frac{1}{a_{2}+\ddots \ddots}} \text { [continued fraction] } \\
\quad ?(x)=\frac{1}{2^{a_{1}-1}}-\frac{1}{2^{a_{1}+a_{2}-1}}+\frac{1}{2^{a_{1}+a_{2}+a_{3}-1}}-\cdots \text { [alternated binary]. }
\end{gathered}
$$

Our generalization, $\Phi_{\alpha, \tau}$, follows the same pattern:

$$
\begin{aligned}
& x=\sum_{r=1}^{\infty} \frac{(\alpha-1)^{r}}{\alpha^{a_{r}}}[(\alpha, \alpha-1) \text {-expansion }] \mapsto \mapsto \\
& \Phi_{\alpha, \tau}(x)=\sum_{r=1}^{\infty} \frac{(\tau-1)^{r}}{\tau^{a_{r}}}[(\tau, \tau-1) \text {-expansion }] .
\end{aligned}
$$

In all the cases, the metrical properties of the real number expansions make the study of the singularity of the functions much easier and allows us to obtain further results on the value of the derivative as we have already shown in [21] and [12]. 


\section{REFERENCES}

1. Patrick Billingsley, Ergodic theory and information, John Wiley \& Sons Inc., New York, 1965. MR MR0192027 (33 \#254)

2. _ Probability and measure, third ed., Wiley Series in Probability and Mathematical Statistics, John Wiley \& Sons Inc., New York, 1995, A Wiley-Interscience Publication. MR MR1324786 (95k:60001)

3. Georges de Rham, Sur une courbe plane, J. Math. Pures Appl. (9) 35 (1956), 25-42. MR MR0090628 (19,842e)

4. A. Denjoy, Sur quelques points de la théorie des fonctions, C. R. Acad. Sci. Paris 194 (1932), $44-46$.

5. — Sur une fonction de Minkowski, C. R. Acad. Sci. Paris 198 (1934), 44-47.

6. Sur une fonction réelle de Minkowski, J. Math. Pures Appl. 17 (1938), 105-151.

7. Roland Girgensohn, Constructing singular functions via Farey fractions, J. Math. Anal. Appl. 203 (1996), no. 1, 127-141. MR MR1412484 (97f:26006)

8. E. Hellinger, Die othogonalinvarianten quadratischer formen von unendlichvielen variabelen, Ph.D. thesis, Göttingen, 1907.

9. Edwin Hewitt and Karl Stromberg, Real and abstract analysis. A modern treatment of the theory of functions of a real variable, Springer-Verlag, New York, 1965. MR MR0188387 (32 \#5826)

10. J. R. Kinney, Note on a singular function of Minkowski, Proc. Amer. Math. Soc. 11 (1960), 788-794. MR MR0130330 (24 \#A194)

11. H. Minkowski, Verhandlungen des III internationalen mathematiker-kongresses in Heidelberg, 1904, Also in Gesammelte Abhandlungen, 1991, vol 2, pp. 50-51 for the? function.

12. J. Paradís, P. Viader, and L. Bibiloni, The derivative of Minkowski's ?(x) function, J. Math. Anal. Appl. 253 (2001), no. 1, 107-125. MR MR1804596 (2002c:11092)

13. G. Ramharter, On Minkowski's singular function, Proc. Amer. Math. Soc. 99 (1987), no. 3, 596-597. MR MR875407 (88c:11013)

14. Sylvester Reese, Some Fourier-Stieltjes coefficients revisited, Proc. Amer. Math. Soc. 105 (1989), no. 2, 384-386. MR MR938913 (89i:42020)

15. A. Rényi, Representations for real numbers and their ergodic properties, Acta Math. Acad. Sci. Hungar 8 (1957), 477-493. MR MR0097374 (20 \#3843)

16. Frigyes Riesz and Béla Sz.-Nagy, Functional analysis, Dover Books on Advanced Mathematics, Dover Publications Inc., New York, 1990, Translated from the second French edition by Leo F. Boron, Reprint of the 1955 original. First published in French in 1952 by the Hungarian Academy of Science. MR MR1068530 (91g:00002)

17. R. Salem, On some singular monotonic functions which are strictly increasing, Trans. Amer. Math. Soc. 53 (1943), 427-439. MR MR0007929 (4,217b)

18. Iekata Shiokawa, Ergodic properties of piecewise linear transformations, Proc. Japan Acad. 46 (1970), 1122-1125. MR MR0289750 (44 \#6938)

19. Lajos Takćs, An increasing continuous singular function, Amer. Math. Monthly 85 (1978), no. 1, 35-37. MR MR481327 (80f:26005)

20. R. F. Tichy and J. Uitz, An extension of Minkowski's singular function, Appl. Math. Lett. 8 (1995), no. 5, 39-46. MR MR1356295 (96i:26005)

21. Pelegrí Viader, Jaume Paradís, and Lluís Bibiloni, A new light on Minkowski's ?(x) function, J. Number Theory 73 (1998), no. 2, 212-227. MR MR1658027 (2000a:11104)

J. Paradís and P. Viader: Departament d'Economia i Empresa, Universitat Pompeu Fabra, Barcelona, Spain. Lluís Bibiloni. Universitat Autònoma de Barcelona, BARCELONA, SPAin

E-mail address: jaume.paradis@upf.edu

E-mail address: pelegri.viader@upf.edu

E-mail address: lluis.bibiloni@uab.es 